\title{
Simulación Estática y Dinámica de un Modelo Físico del Diodo PiN en Carburo de Silicio
}

\author{
Leobardo. Hernández ${ }^{(1)}$, Guillermo. Arzate ${ }^{(1)}$, Zabdiel. Brito ${ }^{(1)}$, Marco. Rodríguez $^{(2)}$ \\ (1) ESIME-C del IPN; Av. Sta. Ana No. 1000, Col. San Fco. Culhuacan. C.P. 04430, DF-México \\ (2) Universidad Autónoma del Carmen, C.P. 24180. Ciudad del Carmen, Campeche-México \\ (email: bilbito_98@yahoo.com, Ihernandezg@ipn.mx)
}

Recibido Nov. 12, 2009; Aceptado Feb. 08, 2010; Versión Final recibida Mar. 22, 2010

\begin{abstract}
Resumen
Este artículo presenta una propuesta de modelado y simulación del diodo PiN en Carburo de Silicio. La propuesta principal de modelado soluciona la ecuación de difusión ambipolar a partir de una aproximación empírica. Mediante la metodología utilizada se obtiene un conjunto de ecuaciones diferenciales que modelan los principales fenómenos físicos asociados al dispositivo semiconductor de potencia. Las ecuaciones implementadas en Pspice modelan en una forma más real el comportamiento de la dinámica de cargas en la región $\mathrm{N}$ - de un diodo PiN en Carburo de Silicio para las fases estáticas y dinámicas. Para la comprobación y validación del modelo desarrollado, se compararon los resultados de simulación con datos experimentales reportados en la literatura, obteniéndose resultados adecuados para aplicaciones de electrónica de potencia.
\end{abstract}

Palabras clave: simulación, modelado, diodo-PiN, carburo de silicio

\section{Static and dynamic simulation of a Physically-Based Model of Silicon Carbide PiN Diode}

\begin{abstract}
This paper presents a method to solve the ambipolar diffusion equation for modeling and simulating the PiN diode in silicon carbide, using an empirical approximation. Through this methodology a set of differential equations that simulate the main physical phenomena associated to the power semiconductor device are obtained. The equations, implemented in Pspice, model in a more actual form the charges behaviour in the $\mathrm{N}$ - region of a PiN diode in silicon carbide for the static and dynamic phases. For the verification and validation of the model, the simulation results were compared with experimental data reported in the literature, obtaining accurate results for application in power electronic.
\end{abstract}

Keywords: simulation, modelling, PiN diode, silicon carbide 


\section{INTRODUCCIÒN}

El Carburo de Silicio ( $\mathrm{SiC}$ ) es uno de los materiales idóneos para reemplazar al silicio en la fabricación de dispositivos de potencia, debido a sus superiores propiedades físicas. Estas propiedades son: una banda prohibida más amplia (2x), una velocidad de saturación de electrones más elevada (2x) y una mayor conductividad térmica (5x) (Zolper, 2005; Singh, 2006; Agarwal, 2006; Holz, 2007). De los distintos dispositivos de potencia que se han desarrollado en SiC (Harada, 2004; Zhao, 2004; Zhang, 2004; Su, 2006), el diodo PiN es una de las estructuras en semiconductores más importantes para desarrollar dispositivos de potencia. Por lo tanto, es de interés el desarrollo de un modelo para diodo $\mathrm{PiN}$ en $\mathrm{SiC}$ que simule correctamente el comportamiento de las diferentes fases de conmutación. La propuesta de modelado está basada en solucionar la ecuación de difusión ambipolar (ADE) a partir de una aproximación empírica dependiente de la longitud de difusión ambipolar. La metodología de modelado permite obtener un conjunto de ecuaciones diferenciales que simulan los principales fenómenos físicos asociados al dispositivo semiconductor en forma continua durante los cálculos, lo que permite un comportamiento mas real de las cargas que se desarrollan en el diodo PiN, las expresiones obtenidas son de fácil implementación en el simulador de circuitos eléctricos Pspice. Para la validación del modelo y por consiguiente de la propuesta de solución de la $A D E$, se compararon los resultados de simulación obtenidos con Pspice con datos experimentales reportados en la literatura.

\section{PRINCIPIO DE MODELADO}

El desarrollo del modelo se basa en la estructura básica de la Fig. 1, en ésta, N- representa la región de bajo dopado, $\left(N_{B}\right) \cdot I_{n(x=0)}, I_{p(x=0)}, I_{n(x=W)}$ e $I_{p(x=W)}$ representan las corrientes inyectadas al diodo, $V_{J 1} y$ $\mathrm{V}_{\mathrm{J} 2}$ representan las caídas de tensión en las uniones, $\mathrm{P}+\mathrm{N}-\mathrm{y} \mathrm{N}-\mathrm{N}+, \mathrm{x}_{\mathrm{L}}$ y $\mathrm{x}_{\mathrm{R}}$ representan la profundidad de formación de las regiones de desérticas. $W=W_{B}-X_{L}-X_{R}$ representa el ancho efectivo de la región $\mathrm{N}$-. Para la obtención del modelo, se parte de la ADE descrita por (1) (Sze, 1981 y Neamen, 2003), donde: $L$ representa la longitud de difusión ambipolar, $D$ se conoce como el coeficiente de difusión ambipolar. Para el desarrollo del modelo se propone que las corrientes de huecos se calculen con la solución particular de la ADE y de la ecuación de transporte descrita por (2) para cada fase de conmutación, las corrientes de electrones se calcularán con la ecuación de Shockley como se observa en (3). En condiciones de polarización aplicada al dispositivo, la región Nexperimenta la inyección y desalojo de cargas $\left(Q_{B}\right)$ las cuales dependen de la solución para $p(x)$, de acuerdo con la Fig.1 el comportamiento de $\mathrm{p}(\mathrm{x})$ es variable, la variación anterior establece una resistencia variable debido al efecto de conductividad por modulación, la expresión para su cálculo está dada por (4). (Sze, 1981 y Neamen, 2003).

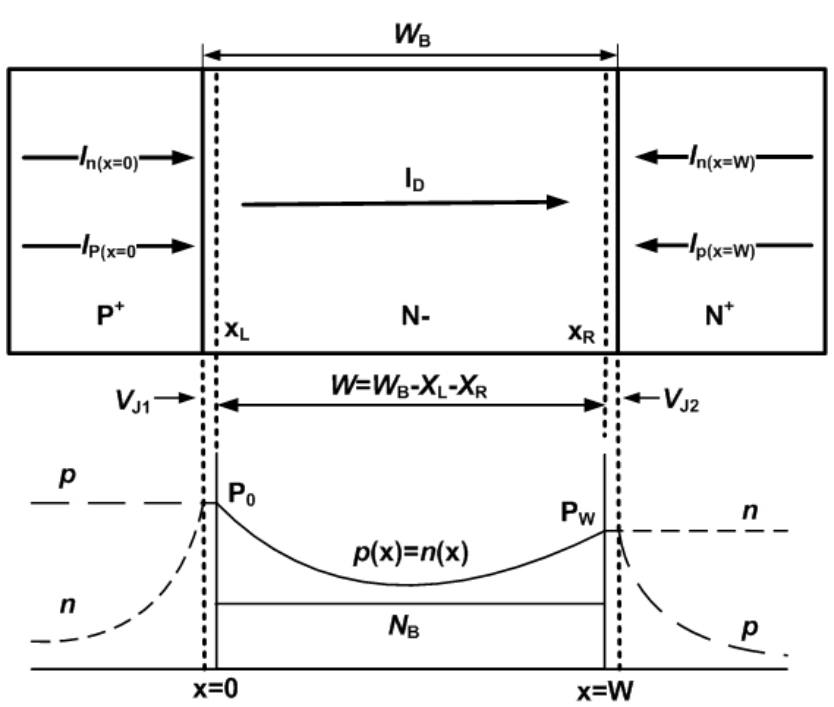

Fig. 1. Estructura básica de diodo PiN.

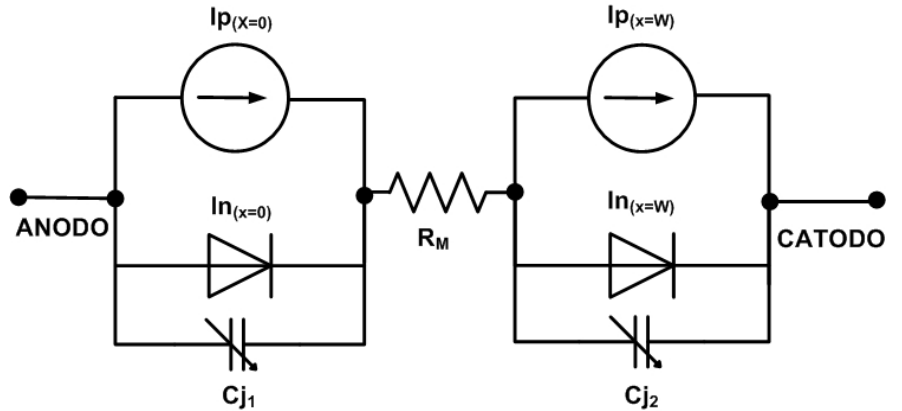

Fig. 2. Equivalente eléctrico simplificado para simulación de diodo PiN 


\section{CIRCUITO EQUIVALENTE ELÉCTRICO}

En la Fig. 2 se muestra el diagrama eléctrico simplificado utilizado para el modelado del diodo. Las fuentes controladas de corriente calculan la componente de huecos a partir de (2), los diodos genéricos calculan las corrientes de electrones a partir de (3), se incorpora al modelo el cálculo de la dinámica de cargas en la región N-, $Q_{B}$ a través de (5).

$$
\begin{aligned}
& \frac{\partial^{2} p(x)}{\partial x^{2}}=\frac{p(x)}{L^{2}}+\frac{1}{D} \frac{\partial p(x)}{\partial t} \\
& I_{p(x)}=\frac{1}{1+b} \cdot I_{T}-q \cdot A \cdot D \frac{\partial p(x)}{\partial x} \\
& I_{n(x=0, x=W)}=I S\left[\exp \left(\frac{V_{j 1, J 2}}{V_{T}}\right)-1\right] \\
& R_{M}=\frac{W^{2}}{\left(\mu_{n}+\mu_{p}\right) \cdot Q_{B}+\mu_{n} Q_{0}} \\
& Q_{B}=q \cdot A \int_{0}^{W} p(x) \cdot d x \\
& p(x, t)=\frac{P_{W} \cdot \sinh \left(\frac{x}{L_{S}}\right)+P_{0} \cdot \sinh \left(\frac{W-x}{L_{S}}\right)}{\sinh \left(\frac{W}{L_{S}}\right)} \\
& p(x, t)=\frac{P_{W} \cdot \sinh \left(\frac{x}{L_{o n}}\right)+P_{0} \cdot \sinh \left(\frac{W-x}{L_{o n}}\right)}{\sinh \left(\frac{W}{L_{o n}}\right)}
\end{aligned}
$$

\section{SOLUCIÒN APROXIMADA DE LA ADE}

Para el estado estático, se cumple que $\partial p(x, t) / \partial t=0$. La solución de (1) esta dada por (6), donde $L_{S}=\sqrt{D \cdot \tau}$ representa la longitud de difusión ambipolar para el estado estático; $P_{0}$ y $P_{w}$ se definen como las concentraciones iniciales en la frontera de las uniones (Grove, 1967 y Baliga, 1996). Para la fase de encendido, se cumple que $\partial p(x, t) / \partial t \neq 0$. Como la ADE no presenta una solución analítica, se utiliza la aproximación empírica: $\partial p(x, t) / \partial t \approx p(x, t) / T$. Con la aproximación propuesta, la solución de

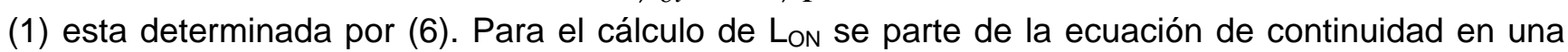
dimensión (Sze, 1981, Neamen, 2003, González, 2009).

$\frac{\partial p}{\partial t}=-\frac{1}{q \cdot A} \cdot \frac{d I_{p}}{d x}-\frac{p}{\tau}$

Integrando entre los limites $\mathrm{x}=0$ y $\mathrm{x}=\mathrm{W}$ y con $\mathrm{Q}_{\mathrm{B}}$, se obtiene la ecuación de control de carga.

$\frac{1}{q \cdot A} \int_{x=0}^{x=w} \frac{d I_{p}}{d x} d x=-\int_{x=0}^{x=w} \frac{p}{\tau} d x-\int_{x=0}^{x=w} \frac{\partial p}{\partial t} d x=I_{p(x=0)}-I_{p(x=W)}=\frac{Q_{B}}{\tau}+\frac{d Q_{B}}{d t}$

Sustituyendo (2), (6) y (8) en (9), se obtiene la expresión final para el cálculo de LoN(t).

$L_{\mathrm{ON}}(t)=\sqrt{\frac{D \cdot Q_{B}}{\frac{Q_{B}}{\tau}+\frac{d Q_{B}}{d t}}}$ 
Para la fase de apagado se propone modelar la distribución de $\mathrm{p}(\mathrm{x}, \mathrm{t})$ en la región $\mathrm{N}$ - por secciones, como se muestra en la Fig. 3. Las ecuaciones desarrolladas que modelan cada una de las secciones propuestas se muestran en (11), (12) y (13). Para que las expresiones propuestas para el apagado simulen correctamente el comportamiento de las cargas $\mathrm{Q}_{B}$ a desalojar de la región $\mathrm{N}-$, se desarrollaron las expresiones analíticas para el cálculo de las tres nuevas longitudes de difusión ambipolar (González, 2009).

$$
\begin{aligned}
& p_{1(x, t)}=P_{L} \operatorname{Cos}\left(\frac{x-x_{L}}{L_{\text {off } 1}}\right) \\
& p_{2(x, t)}=\frac{P_{R} \operatorname{Sinh}\left(\frac{x-x_{L}}{L_{\text {off } 2}}\right)+P_{L} \operatorname{Sinh}\left(\frac{x_{R}-x}{L_{\text {off } 2}}\right)}{\operatorname{Sinh}\left(\frac{x_{R}-x_{L}}{L_{\text {off } 2}}\right)} \\
& p_{3(x, t)}=P_{R} \operatorname{Cos}\left(\frac{x-x_{R}}{L_{\text {off } 3}}\right)
\end{aligned}
$$

Para $L_{\text {off } 1}$ se encuentra de la derivada de (11) evaluada en $x=0$.

$\left.\frac{\partial p_{1(x, t)}}{\partial x}\right|_{x=0}=\frac{P_{L}}{L_{\text {off } 1}} \cdot \operatorname{sen}\left(\frac{x_{L}}{L_{\text {off } 1}}\right)$

Sustituyendo en (14) la relación $P_{0} / P_{L}$ evaluada en $x=0$ de (11), se obtiene la expresión final.

$$
L_{\text {off } 1}=\frac{\sqrt{P_{L}^{2}-P_{0}^{2}}}{\left.\frac{d p_{1}(x, t)}{d x}\right|_{x=0}}
$$

Para $L_{\text {off2 }}$ la corriente total se puede aproximar en forma análoga a la fase de encendido en $x_{L} y x_{R}$, como:

$$
I_{T(x)} \approx I_{p\left(x_{L}, t\right)}-I_{p\left(x_{R}, t\right)}
$$

Sustituyendo (16) en (9), y considerando las corrientes de desplazamiento que se presentan en las regiones desérticas, se obtiene:

$$
I_{p(x L)}-I_{p(x R)}=\frac{Q_{o f f 2}}{\tau}+\frac{d Q_{o f f 2}}{d t}-A \cdot q \cdot P_{L} \cdot \frac{d x_{L}}{d t}+A \cdot q \cdot P_{R} \cdot \frac{d x_{R}}{d t}
$$

Para el cálculo de $I p\left(x_{L}, t\right)$ e $I p\left(x_{R}, t\right)$ se evalúa a (2) en $x_{L}$ y $x_{R}$. Sustituyendo en (17) se obtiene la expresión final.

$$
L_{\text {off } 2}=\sqrt{\frac{D \cdot Q_{\text {off } 2}}{\frac{Q_{\text {off } 2}}{\tau}+\frac{d Q_{\text {off } 2}}{d t}-q \cdot A \cdot P_{L} \frac{d x_{L}}{d t}+q \cdot A \cdot P_{R} \frac{d x_{R}}{d t}}}
$$

Para $L_{\text {off3 }}$ se encuentra la derivada de la ecuación (13) evaluada en $x=W$

$$
\left.\frac{\partial p_{3(x, t)}}{\partial x}\right|_{X=W}=\frac{P_{R}}{L_{\text {off } 3}} \cdot \operatorname{sen}\left(\frac{W-x_{R}}{L_{\text {off } 3}}\right)
$$


Sustituyendo en (19), la relación $P_{W} / P_{R}$ evaluada en $x=W$ de (13). Se obtiene la expresión final

$L_{\text {off } 3}=\frac{\sqrt{P_{R}^{2}-P_{W}^{2}}}{\left.\frac{d p_{3}(x, t)}{d x}\right|_{x=W}}$

$\mathrm{Q}_{\text {off1 }}, \mathrm{Q}_{\text {off2 }}$ y $\mathrm{Q}_{\text {off3 }}$ se calculan con (5) y la $\mathrm{p}(\mathrm{x}, \mathrm{t})$ propuesta en cada sección, el desarrollo a detalle de la obtención de las expresiones (10)-(20) se encuentra en (González, 2009).

\section{RESULTADOS}

En la Fig. 4, se presentan resultados de simulación obtenidos en Pspice para $P_{0}, P_{W}$ y $Q_{B}$. Con $n_{i} \approx 6.7 \times 10^{-11} \mathrm{~cm}^{-3}$ y asumiendo $N_{A} \cdot N_{D}$ a valores típicos de $10^{35} \mathrm{~cm}^{-6}$, el voltaje de encendido teórico es de 2.70V (Baliga, 2005). En los resultados de simulación se comprueba la inyección de cargas a la región $\mathrm{N}$ - a un voltaje de $2.7 \mathrm{~V}$, el resultado anterior corrobora el valor teórico esperado.

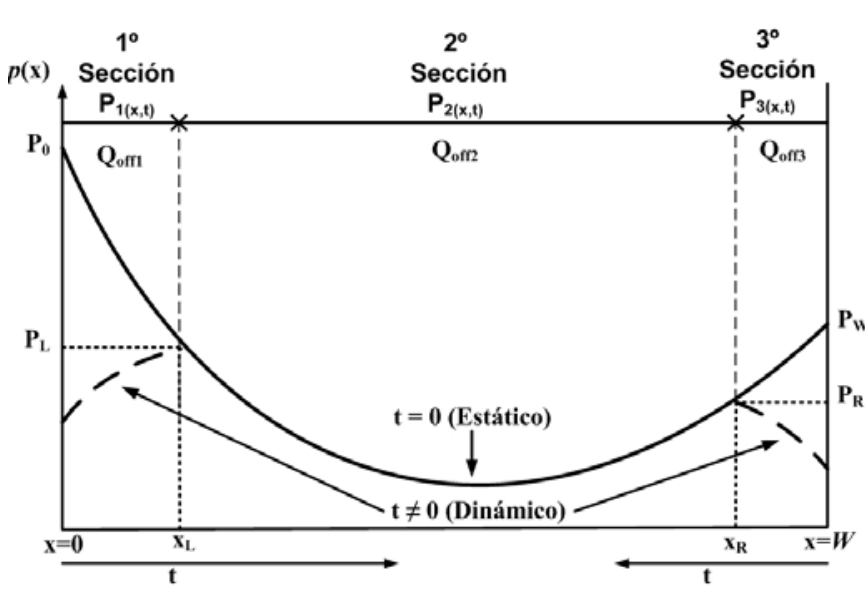

Fig. 3. Modelado por secciones de $p(x)$ en la fase de apagado.

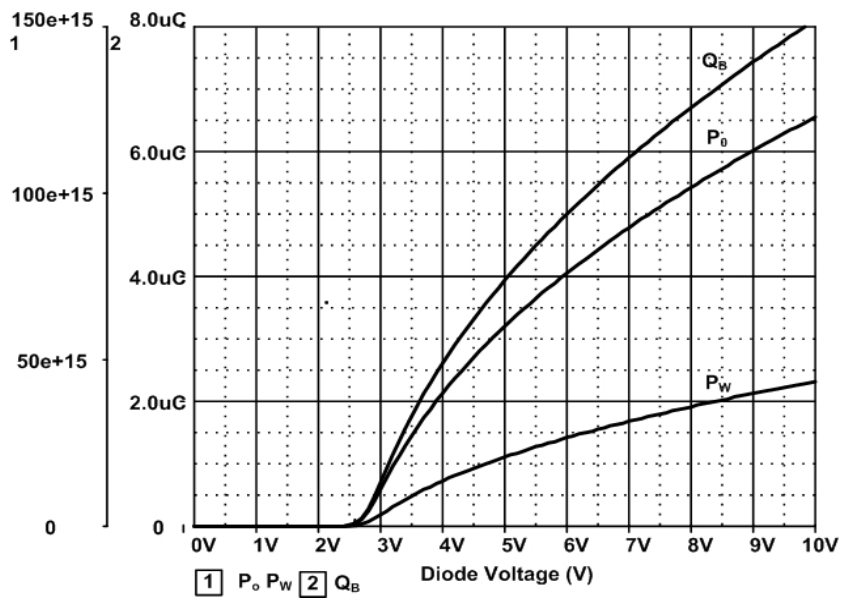

Fig. 4. Resultados de simulación en Pspice para $Q_{B}, P_{0}$ y $P_{W}$.

En la Figura 5, se observa la comparación de resultados de simulación con datos experimentales, reportados en la literatura, para la fase estática (McNutt, 2004). En la Fig. 6, se observa la comparación de resultados de simulación con datos experimentales reportados en las referencias bibliográficas para la fase de apagado (McNutt, 2004).

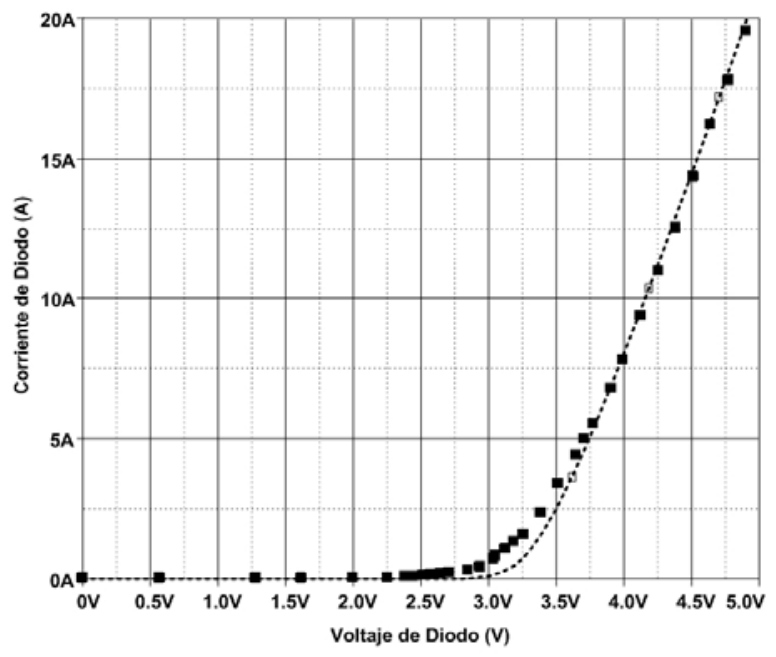

Fig. 5. Simulación (Línea) comparado con experimental (símbolos) para $I_{D}=5 A$. CONCLUSIONES

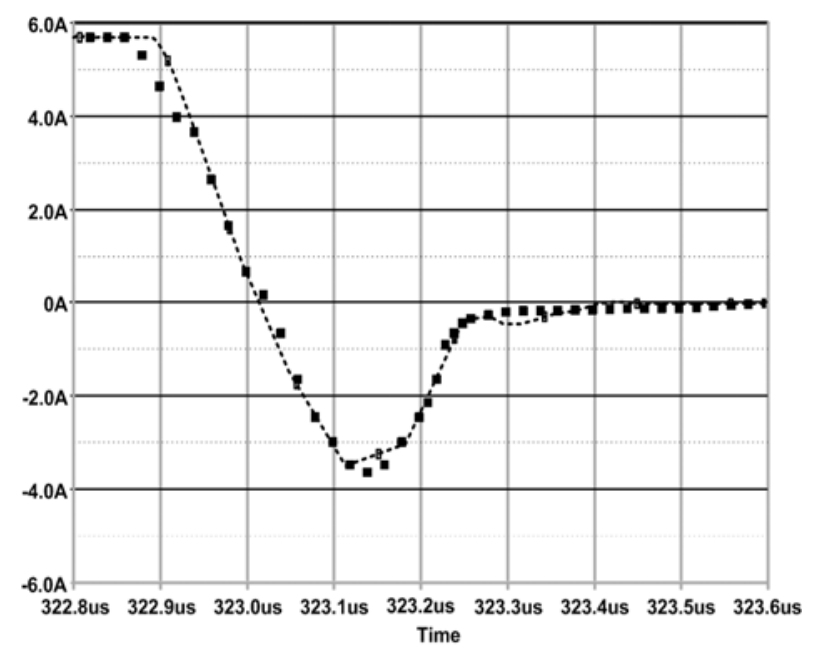

Fig. 6. Simulación (línea) comparados con experimental (símbolos) para $I_{D}=5 \mathrm{~A}$. 
Se presentó una metodología de solución para la ecuación de difusión ambipolar (ADE), basada en la propuesta de aproximación empírica dependiente de la longitud de difusión ambipolar. La metodología de solución permite obtener cálculos continuos durante todo el proceso de simulación, lográndose un modelado más real del comportamiento de las cargas en la estructura de diodo como se observo en las comparaciones con datos experimentales. El modelo matemático desarrollado se implementó en el simulador de circuitos eléctricos Pspice. Para la validación del modelo se tomaron en cuenta datos experimentales reportados en referencias bibliográficas. El modelo fue simulado para una corriente de conducción máxima de 5A. Finalmente el modelo es de fácil implementación y toma en cuenta los principales fenómenos que se presentan en el carburo de silicio.

\section{REFERENCIAS}

Agarwal, A. y otros 7 autores, Progress in Silicon Carbide Power Devices, IEEE Devices Research Conference, 26 al 28 Junio (2006).

Baliga, B. J. Power Semiconductor Devices, $1^{\mathrm{a}}$ edición, PWS Publishing Company, USA (1996).

Baliga, B. J. Silicon Carbide Power Devices, $1^{\text {a }}$ edición, World Scientific, USA (2005).

Grove, A. S. Physics and Technology of Semiconductor Devices, $1^{\text {a }}$ edición, John Wiley \& Sons, USA (1967).

Harada, S. y otros 5 autores, $8.5-\mathrm{m} \Omega \mathrm{cm}^{2}$ 600V Double-Epitaxial MOSFETs in 4H-SiC, Transaction Electron Device Letter, Vol 25, pp 292-294 (2004).

González, L. Sánchez, A. Rodríguez, M. Anguiano, T. Solución aproximada de la ADE basada en la longitud de difusión aplicada en la simulación del diodo SiC PiN, The Mexican Journal of Electromechanical Engineering, ISSN 1665-0654, Vol 13, pp 55-62, México (2009).

Holz, M.; Hultsch, T.; Rupp, R. "Reliability considerations for recent Infineon SiC diode releases", Microelectronics Reliability, Vol 47, pp 1741-1745, (2007).

McNutt, Ty. Y otros 5 autores, Silicon Carbide PiN and Merged PiN Schottky Power Diode Models Implemented in the Saber Circuit Simulator, Transaction Power Electronic, IEEE, Vol 19, pp 573-581 (2004).

Neamen, D. A. Semiconductor Physics and Devices, $3^{a}$ edición, McGraw Hill, USA (2003).

Singh, S. Reliability and Performance Limitations in SiC Power Devices, Microelectronics Reliability Vol 46, pp 713-730 (2006).

Su, M. y otros 6 autores, 430-V 12.4- $\mathrm{m} \Omega \mathrm{cm}^{2}$ Normally OFF 4H-SiC Lateral JFET, Electron Devices Letter, Vol 27, pp 834-836 (2006).

Sze, S. Physics of Semiconductor Devices, $1^{\text {a }}$ edición, John Wiley \& Sons, USA (1981).

Zhao, J.H. y otros 4 autores, Demonstration of $1789 \mathrm{~V}, 6.68 \mathrm{~m} \Omega-\mathrm{cm}^{2} 4 \mathrm{H}$-SiC Merged-PiN-Schottky Diodes, Electronics Letter, Vol 40, Marzo (2004).

Zhang, J. y otros 3 autores, Demonstration of First $9.2 \mathrm{kV} 4 \mathrm{H}$-SiC Bipolar Junction Transistor, Electronics Letter, Vol 40, Octubre (2004).

Zolper, J.C. Emerging Silicon Carbide Power Electronics Components, IEEE Applied Power Electronics Conference and Exposition, 19 al 23 de Febrero (2005). 